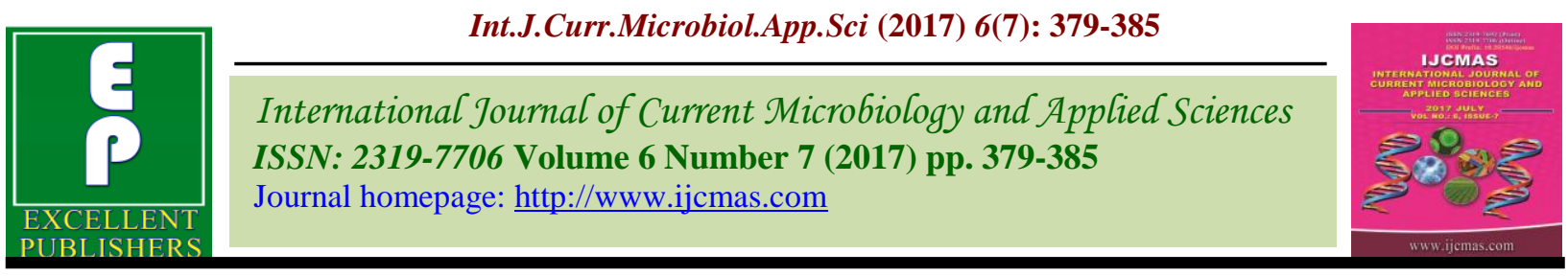

Original Research Article

https://doi.org/10.20546/ijcmas.2017.607.045

\title{
Improvement in Egg Production of PD 3 Chicken Line with Histopathological Conditions of the Jejunum up on Supplementation of Fermented Yeast Culture during and Post Summer Season
}

\author{
N. Anand Laxmi", M. Shanmugam, M.R. Reddy and R.K. Mahapatra \\ ICAR- Directorate of Poultry Research, Rajendranagar, Hyderabad, A.P., India \\ *Corresponding author
}

A B S T R A C T

During summer, under high ambient temperature conditions, the thermoregulatory system of the body adjusts a variety of physiological mechanisms through a combination of heat exchange and evaporative heat loss to attain a balance between the heat produced within the body and the heat lost to the environment. In chickens sweat glands are absent making it more vulnerable for dissipation of heat and reducing stress. Leptin affects energy homeostasis by decreasing food intake and by upregulating fatty acid oxidation. In chicken leptin and ghrelin are known as anorexigenic hormones. Effects of yeast products on production and their mode of action in monogastrics have been reported

Keywords

Summer,

Layers,

Fermented yeast

culture,

Leptin,

Ghrelin,

MDA,

Cholesterol.

Article Info

Accepted:

04 June 2017

Available Online:

10 July 2017 in poultry. It is reported that yeast products affect nutrient digestibility, growth and immune system. Fermented yeast culture contains viable cells, cell wall components, metabolites, and the media on which the yeast cells were grown. No study has been carried out on endocrinological and histopathological studies with supplementation of fermented yeast culture in chicken and their relation with egg production, especially during summer season. This study was carried out to determine pattern of leptin and ghrelin secretion among other oxidative markers in PD 3 chicken line (Dahlem Red) with histopathological conditions in the intestine, when subjected to natural heat stress during summer season. During summer the temperature ranged between $26^{\circ} \mathrm{C}$ and $36.9^{\circ} \mathrm{C}$ and post summer season it ranged between $29^{\circ} \mathrm{C}$ and $32^{\circ} \mathrm{C}$. A hundred number of 16 week old Dahlem Red (PD-3) breed of chickens whose body weight ranged between $850-950 \mathrm{~g}$ were selected and randomly allotted to two groups of fifty birds each. The experiment was continued from 16-32 weeks of age. After eight weeks (April-May) of summer season, experiment was continued till the birds attained 32 weeks of age. The two groups were Control and Treatment groups. The treatment group received $1.25 \mathrm{~g}$ of fermented yeast culture (Saccharomyces cerevisiae) commercial product per $\mathrm{kg}$ feed. Blood samples were collected at weekly intervals, plasma was separated and analyzed for Leptin, Ghrelin hormones, plasma protein carbonyls, MDA, cholesterol and free fatty acids. Fortnightly body weight and feed intake of the birds were recorded. Egg production and egg weight were also recorded. Based on these observations, feed conversion ratio (FCR) was also calculated. Supplementation of fermented yeast culture decreased the concentration of plasma Leptin and Ghrelin $(\mathrm{P}<0.01)$, Cholesterol $(\mathrm{P}<0.01)$ and MDA $(\mathrm{P}<0.01)$ during summer season and feed intake of birds during post summer season. It was observed that the egg production increased from 26-31 weeks of age and FCR was significantly less $(\mathrm{P}<0.05)$ in the supplemented group. It was observed that fertility and hatchability of eggs was more for the supplemented group. In conclusion providing fermented yeast culture to the sexually maturing hens during peri and post summer period improved feed efficiency, egg production with no improvement in the morphology of the jejunum. However the severity of necrosis decreased from medium to mild stage in both the groups from pre to post laying period.

\section{Introduction}

Hyperthermia is defined as an elevated body temperature due to failed thermoregulation. During hyperthermia, core body temperature increases due to an inability to dissipate heat(Fuquay, 1981). During the initial period of heat stress heat shock proteins assist in 
folding of the membrane proteins and stabilize membrane lipids for maintaining the homeostasis of the cell integrity. When the organism gets acclimatized to elevated temperature, it leads to decrease in the sensitivity towards stress and the response to the stress is delayed. One of the best described effects of oxidative stress-generated ROS on cells is the oxidation of membrane lipids. The susceptibility of lipids to oxidation is determined by its composition and degree of saturation. Depending on the severity of heat stress, the stress signals are activated and which in turn determine the response of the organism to stress. Chronic heat stress leads to depletion of antioxidant reserves. HS has been a great concern to the poultry producers, especially in the tropical regions of the world, during summer season. Chronic HS is categorized as cyclic chronic heat stress, which refers to a limited period of exposure to elevated temperature followed by comfortable temperature for the rest of the day. The consequences of heat stress on physiological functions are numerous, leading to impairment in reproductive and endocrine functions, disruption in structure and function of intestinal epithelium. Azad et al., (2013) observed that in chronic heat exposed chickens, ROS production increased and was found until d9 of HS and not thereafter, which means that chickens became acclimatized to the heat stress. In $18 \mathrm{~h}$ heat stressed chickens, plasma and mitochondrial malondialdehyde (MDA) was 2.1- and 2.7-fold higher (Mujahid et al., 2007). Even though the role of appetite regulating hormones like Leptin, Ghrelin on feed intake, functions of liver is known (Song et al., 2012), their role on physiological and metabolic functions of chickens experiencing heat stress during summer season is lacking. In addition to being a primary organ for absorption and digestion of nutrients, the GIT also contains a diverse set of microbes, which aid in metabolic processes as well as host defense mechanisms. Impairment of small intestinal function can occur with or without morphological changes to the epithelium. Functional integrity of the intestine is dependent upon coordinated regulation of the mucus layer, tight junctions, epithelial cells, as well as the enteric immune system (Groschwitz and Hogan, 2009). Nutrient absorption optimization and post-absorptive metabolism changes have been observed in heat stress poultry (Garriga et al., 2006). Appetite regulating peptides such as ghrelin, cholecystokinin, and glucagon-like peptide 1 released from digestive tract act on the satiety centres in the hypothalamus, which ultimately results in reduced nutrient absorption from the intestine (de Lartigue et al., 2011). Since there is a strong relation between mentioned gut hormones, feed intake, and can be modulated by heat stress. This study was primarily taken up for evaluating the effect of supplementation of fermented yeast culture in maintaining morphology of the jejunum portion of the gut on the level of plasma hormones, production parameters under the influence of higher ambient temperature during and post summer season.

\section{Materials and Methods}

Study for the summer period was carried out from the last week of April-last week of June (eight weeks) in the poultry farm, Directorate of Poultry Research, situated in Rajendranagar, Hyderabad. The month of April and May is characterized by gradually rising daily high temperatures, with daily high around $37^{\circ} \mathrm{C}$ throughout the month, exceeding $41^{\circ} \mathrm{C}$ or dropping below $34^{\circ} \mathrm{C}$ only one day in ten. Birds with uniform body weight ranging between 850-950g were taken for the present study. The birds were divided in to two groups, one served as control and the other served as treatment/supplemented group. Each group contained 50 birds with five birds in each replicate. The supplemented group was provided with fermented yeast culture 
(Saccharomyces cerevisiae) @ 1.25g/kg feed. The experiment was initiated when the birds were 16 weeks of age and by the end of summer, the age of the birds was 24 weeks. The experiment was carried out during post summer period also, which coincided with the laying period of the hens. The experiment continued till the birds attained 32 weeks of age. Fermented yeast culture @ $1.25 \mathrm{~g} / \mathrm{kg}$ was supplemented during and also during post summer period. Blood samples were collected at fortnight intervals, from pectoral vein during summer season only.

The samples were centrifuged at $3000 \mathrm{rpm}$ for $15 \mathrm{~min}$ and analysed for plasma hormones leptin and ghrelin and other parameters cholesterol and MDA. The hormones were estimated with the help of commercially available kits. Plasma MDA was analyzed using the standard method of MDA assay which was based on the reaction of MDA with thiobarbituric acid (TBA); forming an MDA-TBA2 adduct that absorbs strongly at $532 \mathrm{~nm}$. Cholesterol was estimated with the help of commercially available kit purchased from Bio Assay Systems, CA 94545, USA. Body weight was recorded at fortnight interval.

Weekly feed intake was estimated. In the laying period during post summer period, eggs were collected daily and weight was recorded. The birds (5 nos. from each group) were sacrificed during first and last week of summer period and at the end of the laying period, jejunum portion of the digestive tract (from the pancreatic loop to Meckel's diverticulum) was separated, feed particles were removed gently by rinsing in normal saline solution. They were fixed immediately in $10 \%$ formalin for histopathological studies. Samples were processed, paraffin embedded and cut at $5 \mathrm{u}$ thickness. The sections were stained with hematoxylin-eosin. The slides were observed at $20 \mathrm{x}$ magnification, under light microscope. Approval was taken from Institutional Animal Ethics committee for techniques involved in conducting the present study.

\section{Results and Discussion}

The temperature during summer period varied between $29^{\circ} \mathrm{C}-39.6^{\circ} \mathrm{C}$. The circulatory level of plasma leptin, and ghrelin were significantly high $(\mathrm{P}<0.01)$ in the control when compared with the treatment group. In the treatment and control groups, within the group, the highest concentration of the hormones was observed on d21 (Table 1). Higher concentration of anorexigenic hormones did not result in decrease in body weight or feed intake and difference between these parameters was not significant between the groups. Later by $\mathrm{d} 49$ or by the end of summer, the level of plasma leptin and ghrelin in the control group decreased, but, the difference between the level of respective hormones between the groups was still significant $(\mathrm{P}<0.05)$.

The hypothalamic and gastrointestinal tract peptides like ghrelin are involved in appetite regulation in laying hens exposed to heat stress (Song et al., 2012). It appeared that birds of control group were not acclimatized by the end of the summer period. Similar trend was observed with plasma malondialdehyde (MDA) levels (Table 2, $\mathrm{P}<0.01)$. Plasma MDA levels indicate the oxidative state of the lipids. It is known that fermented yeast culture reduces MDA levels (Matur et al., 2011). Hence in the present study also, supplementation of fermented yeast culture, reduced plasma MDA level, indicating protective effect of supplementation on lipid peroxidation or in amelioration of oxidative stress. At the end of the experiment, level of plasma MDA compared between the groups was not significant indicating decrease in lipid peroxidation in the control group (Table 2). It 
is known that heat stress, increases plasma cholesterol level, in the present study also, the level of circulatory cholesterol was higher in the control group. The rise in the concentration of cholesterol was observed on d21 of the experiment and continued till the end of the study period. The concentration did not decrease by the end of summer period in the control group, where as in the treatment group, supplementation of fermented yeast culture decreased the level of plasma cholesterol (Table 2, $\mathrm{P}<0.01$ ) when compared with the control group. Exposure to heat enhances ROS production and induces oxidative stress, which can lead to cytotoxicity (Bernabucci et al., 2002) and lipid peroxidation (Mujahid et al., 2007).

Table.1 Concentration of plasma leptin and ghrelin in control and supplemented group of PD 3 chicken line (Dehlam Red) during summer season

\begin{tabular}{|l|l|l|l|l|}
\hline Days & \multicolumn{2}{|l|}{ Leptin $(\mathrm{ng} / \mathrm{ml})$} & \multicolumn{2}{l|}{ Ghrelin $(\mathrm{pg} / \mathrm{ml})$} \\
\hline & CONTROL & TREATMENT & CONTROL & TREATMENT \\
\hline 7 & $\begin{array}{l}1.67^{* *} \\
\pm 0.12\end{array}$ & $1.23 \pm 0.09$ & $60.23^{*} \pm 2.30$ & $52.10 \pm 2.10$ \\
\hline 21 & $1.85^{* *} \pm 0.15$ & $1.26 \pm 0.09$ & $80.57^{* *} \pm 1.58$ & $64.23 \pm 2.54$ \\
\hline 35 & $1.79^{* *} \pm 0.11$ & $1.10 \pm 0.08$ & $65.70^{*} \pm 2.01$ & $50.60 \pm 1.98$ \\
\hline 49 & $1.54^{*} \pm 0.09$ & $1.23 \pm 0.12$ & $58.23^{*} \pm 1.62$ & $49.32 \pm 2.05$ \\
\hline
\end{tabular}

Table.2 Concentration of plasma cholesterol and MDA in control and supplemented groups of PD 3 chicken line (Dehlam Red) during summer season

\begin{tabular}{|c|c|c|c|c|}
\hline & Cholesterol $(\mathrm{mg} / \mathrm{dl})$ & MDA $(\mathrm{uM} / \mathrm{ml})$ \\
\hline Days & CONTROL & TREATMENT & CONTROL & TREATMENT \\
\hline 7 & $212^{*} \pm 10.23$ & $180 \pm 9.24$ & $140 * * \pm 8.54$ & $68 \pm 3.21$ \\
\hline 21 & $220^{*} \pm 9.56$ & $155 \pm 8.52$ & $135^{* *} \pm 7.89$ & $65 \pm 1.58$ \\
\hline 35 & $230^{*} \pm 8.45$ & $140 \pm 10.23$ & $91^{* *} \pm 5.89$ & $58 \pm 2.03$ \\
\hline 49 & $235^{*} \pm 10.45$ & $135 \pm 9.85$ & $65 \pm 2.35$ & $59 \pm 1.09$ \\
\hline
\end{tabular}

Table.3 Comparison of percentage of egg production Potential post summer season

\begin{tabular}{|c|c|c|}
\hline WEEKS & CONTROL & TREATMENT \\
\hline $\mathbf{2 4}$ & $\mathbf{3 6 . 5}$ & $\mathbf{3 4 . 8}$ \\
\hline $\mathbf{2 5}$ & $\mathbf{4 3 . 7}$ & $\mathbf{4 5 . 8}$ \\
\hline $\mathbf{2 6}$ & $\mathbf{4 7 . 6}$ & $\mathbf{5 0 . 6}$ \\
\hline $\mathbf{2 7}$ & $\mathbf{4 7 . 1}$ & $\mathbf{5 2 . 1}$ \\
\hline $\mathbf{2 8}$ & $\mathbf{4 8 . 6}$ & $\mathbf{5 3 . 7}$ \\
\hline $\mathbf{2 9}$ & $\mathbf{5 0 . 7}$ & $\mathbf{5 5 . 5}$ \\
\hline $\mathbf{3 0}$ & $\mathbf{5 4 . 1}$ & $\mathbf{5 8 . 2}$ \\
\hline $\mathbf{3 1}$ & $\mathbf{5 2 . 9}$ & $\mathbf{5 5 . 1}$ \\
\hline $\mathbf{3 2}$ & $\mathbf{5 4 . 0}$ & $\mathbf{5 6 . 7}$ \\
\hline
\end{tabular}

Attainment of 50\% egg production potential earlier in treatment group Egg production was more in the treatment group (26-32 weeks) 
Table.4 Percentage of fertility and hatchability

\begin{tabular}{|l|l|l|l|}
\hline 28-29 weeks & $\begin{array}{l}\text { Egg set } \\
\text { (nos.) }\end{array}$ & Fertility & Hatchability \\
\hline CONTROL & 210 & 79 & 92 \\
\hline TREATMENT & 252 & 86 & 98 \\
\hline 30-31 weeks & & & \\
\hline CONTROL & 216 & 82 & 90.1 \\
\hline TREATMENT & 230 & 88 & 98.5 \\
\hline
\end{tabular}

Fig.1 a) Histology of the of the jejunum (normal villi) b) Mild to medium necrosis in jejunum portion of the gut; Fusion and thickening of villi.

Observed at 20x magnification under light microscope

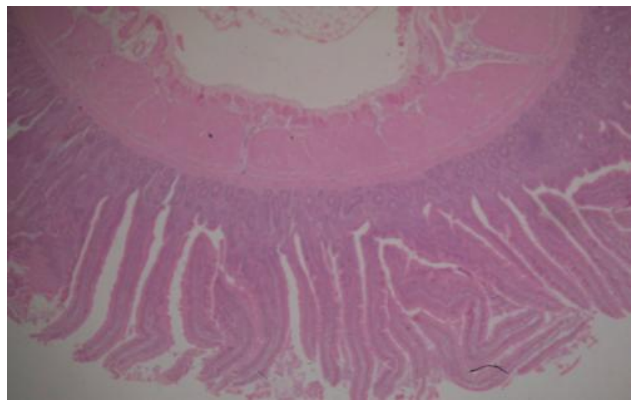

Hypercholesterolemia is caused by hyperactivity of the adrenal gland (Siegel, 1995). It is known that during heat stress, ambient temperature increases level of plasma cortisol, which is indicative of hyperactivity of adrenal gland. In the present study supplementation of fermented yeast culture, might have decreased hyper activity of adrenals, which in turn decreased plasma cholesterol during summer. Similar reports are available in layers and broilers (ElHusseiny et al., 2008). Antioxidant systems are important with regard to the scavenging of free radicals and their metabolic products, as well as in the maintenance of normal cellular physiology, via the restoration of various depleted antioxidants in stressed poultry (Halliwell and Gutteridge, 1989). Yeast culture or its products are also known to play an antioxidant role, preventing lipid peroxidation. Several studies reported that addition of yeast or yeast culture products to diets resulted with better feed efficiency

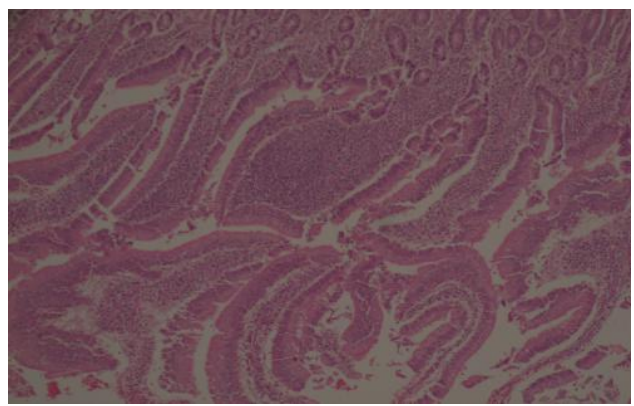

(Tangendjaja and Yoon, 2002), increased egg weight (Yalcin et al., 2008) and improved internal egg quality (Miles and Bootwalla, 1991) in hens. Addition of yeast culture products to hen diets improved feed efficiency (Tangendjaja and Yoon, 2002). In the present study also, it was observed that supplementation improved feed efficiency significantly (4.13vs3.22, $\mathrm{P}<0.05)$. Others have reported that yeast products affect nutrient digestibility (Shin et al., 2005) and intestinal mucosal development (Zhang et al., 2005). The difference in the body weight and egg weight between the groups was not significant during post summer season. It was observed that supplementation decreased feed conversion ratio, but did not affect the morphology of the jejunum portion of the intestine. Inclusion of $\mathrm{YC}(2.5 \mathrm{~g} / \mathrm{kg})$ in broilers decreased the villus height to crypt depth ratio (VCR) in jejunum but increased in duodenum (Gao et al., 2008). A mild to medium necrosis (Figure 1) was observed in 
the histological sections of the jejunum with fusion of villi. Later on occurrence of villi necrosis was decreased in both the groups with the decrease in ambient temperature. Intestinal villi had more desquamation, mostly located at the tip. Heat stress might have caused these effects, but supplementation of FYC did not improve the morphology of the jejunum as observed in the histological sections. The attainment of $50 \%$ egg production potential was earlier in the treatment group, and egg production was more from 26-31 weeks when compared with the control group (Table 3). The decrease in egg production potential as observed for the control group may be due to the presence of mild necrotic condition in the jejunum, and in addition to it the absence of supplement might have led to less absorption of nutrients from the digestive tract. The result with respect to fertility and hatchability of the eggs was observed to be higher for the treatment group during post summer season (Table 4). The values of different plasma parameters when compared between control and treatment groups, it appeared that birds of control group were under chronic cyclic heat stress. At the end of study period, during summer season, from the results on different parameters, it appeared that birds of control group were not getting acclimatized to heat stress/high ambient temperature, the results with respect to egg production, fertility and hatchability parameters for post summer period were not at par with the supplemented group.

In conclusion it can be said that supplementation of fermented yeast culture to layers@1.25g/kg, increased egg production, fertility, hatchability parameters during the post summer season, by decreasing concentration of plasma hormones, MDA and cholesterol of PD 3 chicken line with mild to medium necrotic conditions in the jejunum during summer season. Supplementation did not bring about differential effect on the morphology or necrotic condition of the jejunum, when compared between the groups. Hence supplementation of fermented yeast culture may prove to be beneficial.

\section{Acknowledgements}

Acknowledgements are due to Indian Council of Agricultural Research, New Delhi, for providing funds for carrying out the present research work.

\section{References}

Azad, M.K.A., M. Kikusato, I. Zulkifi and Toyomizu M. 2013. Electrolysed reduced water decreases reactive oxygen speciesinduced oxidative damage to skeletal muscle and improves performance in broiler chickens exposed to medium-term chronic heat stress. British Poultry Science. 54:503-509.

Bernabucci, U., B. Ronchi, N. Lacetera and Nardone, A. 2002. Markers of oxidative status in plasma and erythrocytes of transition dairy cows during hot season. Journal of. Dairy Science. 85: 2173-2179.

Gao, J., H. J. Zhang, S. H. Yu, S. G. Wu, I. Yoon, J. Quigley, Y. P. Gao and Qi, G. H. 2008, Effects of Yeast Culture in Broiler

Diets on Performance and Immunomodulatory Functions. Poultry Science. 87: 1377-1384.

Garriga, C., R.R. Hunter, C. Amat, J.M. Planas, M. A. Mitchell and Moreto, M. 2006, Heat stress increases apical glucose transport in the chicken jejunum. American Journal of Physiology.Regulatory, Integrative Comparative. Physiology. 290: R195-201.

Geraert, P. A., J. C. F. Padilha and Guillaumin S. 1996, Metabolic and endocrine changes induced by chronic heat exposure in broiler chickens: Growth performance, body composition and energy retention. British Journal of Nutrition. 75:195-204.

Groschwitz, K.R. and Hogan, S.P. 2009, Intestinal Barrier Function: Molecular 
Regulation and Disease Pathogenesis. The Journal of Allergy and Clinical Immunology. 124: 3-22.

Halliwell, B. and Gutteridge, J. M. C. 1989, Lipid peroxidation: a radical chain reaction. In: Free radicals in biology and medicine. 2nd Ed. New York: Oxford University Press. pp. 188-218.

El-Husseiny O. M., A. G. Abdallah and AbdelLatif K.O. 2008, the influence of biological feed additives on broiler performance. International Journal of Poultry Science. 7:862-871.

De Lartigue, G., C.B. de La Serre and Raybould H.E. 2011, vagal afferent neurons in high fat diet-induced obesity; intestinal microflora, gut inflammation and cholecystokinin. Physiology and Behavior. 105:100-105

Matur, E., E. Ergul, I. Akyazi, E. Eraslan, G. Inal, S. Bilgic and Demircan H. 2011, British Journal of Poultry Science 52:541550.

Miles, R.D. and Bootwalla S.M. 1991, Directfed microbials in animal production 'avian'. In direct-fed microbials in animal production-a review of the literature. National Feed Ingredients Association, West Des Moines, IA. P. 117-146.

Mujahid, A., N. R. Pumford, W. Bottje, K. Nakagawa, T. Miyaza-wa Y. Akiba, and Toyomizu M. 2007, Mitochondrial oxidative damage in chicken skeletal muscle induced by acute heat stress. Poultry Science. 44:439-445

Shin, Y. W., J. G. Kim, and Whang, K.Y. 2005,
Effect of supplemental mixed yeast culture and antibiotics on nitrogen balance of weaned pigs. Journal of Animal Science. 83(Suppl. 1):34. (Abstr.)

Siegel, H. S. 1995, Stress, strains and resistance. British Poultry Science. 36:322.

Song, Z., L. Liu, A. Sheikhahmadi, H. Jiao and Lin, H. 2012, Effect of heat exposure on gene expression of feed intake regulatory peptides in laying hens. Journal of Biomedicine and Biotechnology. doi: 10.1155/2012/484869.

Tangendjaja, B. and Yoon, I. 2002. Effect of yeast culture on egg production and mortality in layer chickens. In Poultry Science Association 91st Annual Meeting Abstracts. August 11-14, Newark, DE. Abstract No: 380. Pp. 89.

Yalçın, S., B. Özsoy, H. Erol and Yalçın, S.2008, Yeast culture supplementation to laying hen diets containing soybean meal or sunflower seed meal and its effect on performance, egg quality traits and blood chemistry. Journal of Applied Poultry Research. 17: 229-236.

Zhang, A. W., B. D. Lee, S. K. Lee, K. W. Lee, G.H. An, K.B. Song and Lee, C. H. 2005, Effects of yeast (Saccharomyces cerevisiae) cell components on growth performance, meat quality, and ileal mucosa development of broiler chicks. Poultry Science. 84:1015-1021.

\section{How to cite this article:}

Anand Laxmi, N., M. Shanmugam, M.R. Reddy and Mahapatra, R.K. 2017. Improvement in Egg Production of PD 3 Chicken Line with Histopathological Conditions of the Jejunum up on Supplementation of Fermented Yeast Culture during and Post Summer Season. Int.J.Curr.Microbiol.App.Sci. 6(7): 379-385. doi: https://doi.org/10.20546/ijcmas.2017.607.045 